\title{
Serratia marcessens infection presenting with papillovesicular rash similar to varicella zoster infection: a case report
}

\author{
Aysenur Bahadir, Erol Erduran \\ Department of Pediatric Hematology-Oncology, Karadeniz Technical University Faculty of Medicine, Trabzon, Turkey
}

\begin{abstract}
According to the literature, skin manifestations related to Serratia marcessens infections are rarely seen, and observed mostly in immunosuppressed adult patients. Cellulitis, abscess, granulomataus lesions have been reported as skin manifestations of Serratia infections. In our 2 cases with leukemia, papillovesicular rashes were observed resembling those of varicella zoster infection. Serratia marcessens was grown on blood cultures of patients susceptible to meropenem. The patients recovered from the rashes rapidly after treatment. Based on the absence of similar case reports in the literature, we report these two pediatric cases to emphasize that Serratia marcessens infections can present with papillovesicular rash similar to that seen in varicella zoster infections.
\end{abstract}

Key words: Catheter; leukemia; papillovesicular rash; serratia, varicella.

Cerratia marcessens is a gram-negative, opportu$\checkmark$ nistic and nosocomial pathogen belonging to the Enterobacterieae family. It is often found in intensive care units, and most importantly grown in the digestive, respiratory and urinary systems, and newborn perineums and health workers. The incidence of $S$. marcessens-derived nosocomial infections is only 1-2\%; while the main sources of risk for Serratia infections in hospitals for bacteremia/sepsis are intravenous, intraperitoneal and urinary catheters $[1,2]$.

Cutaneous manifestations of $S$. marcessens infections have been reported as ulcer, abscess, granulomatous and nodular skin lesions [2]. S. marcessens infection-related cutaneous manifestations in pediatric population are rarely reported in the medical literature. Herein, we report two pediatric cases presenting with papillovesicular rash caused by $S$. marcessens but misdiagnosed as varicella zoster virus $(\mathrm{VZV})$ infections.

\section{CASE REPORTS}

Case 1 - A three-year-old female patient was diagnosed with low-risk acute lymphoblastic leukaemia (ALL) in our pediatric hematology clinic, and her treatment was initiated according to the St. Jude

Received: September 23, 2014 Accepted: December 08, 2014 Online: April 24, 2015

Correspondence: Dr. Aysenur BAHADIR. Karadeniz Teknik Universitesi Tip Fakultesi, Pediatrik Hematoloji-Onkoloji Bilim Dali, Trabzon, Turkey. 
Total XV chemotherapy protocol. A central venous catheter (CVC) was placed on the 5. day of her hospitalization. Neutropenic sepsis developed on the 5. day of her hospitalization while the patient was on chemotherapy. Blood culture was obtained from the patient, and the patient was put on $100 \mathrm{mg} / \mathrm{kg}$ of cefepime and $15 \mathrm{mg} / \mathrm{kg}$ of amikacin therapy. Growth of Gram-negative bacilli was observed in her blood culture, and the patient developed antibiotic-resistant fever. As a consequence, cefepime treatment was discontinued on day 2 , and $120 \mathrm{mg} / \mathrm{kg}$ of meropenem treatment was started. Growth of meropenem-sensitive $S$. marcessens was observed in both CVC and peripheral blood cultures. On the third day of antibiotherapy, vesiculobullous rashes originating from the dorsum of the hand and abdomen developed which spred to the scalp the next day. The lesions were not itchy, and didn't invade the mucosa.

A sample was tested for VZV IgM based on the resemblance of the lesions to those of a VZV infection, and acyclovir $(30 \mathrm{mg} / \mathrm{kg} / \mathrm{d})$ treatment was started. After the $48^{\text {th }}$ dose, the patient's body temperature started to decrease, returning to a normal level during the follow-up period. Within 3-5 days rashes diminished and the patient fully recovered. When VZV IgM test results became negative, acyclovir treatment was discontinued. However, development of a necrotizing abscess in the gluteal region, and nodular skin lesions on the arms and legs necessitated maintenance of vancomycin $(40 \mathrm{mg} /$ $\mathrm{kg}$ ) treatment. CVC was removed after $S$. marcessens growth on CVC samples was twice than that observed in the CVC blood culture. Amikacin, and meropenem were discontinued on day 19 after absence of bacterial growth in peripheral blood cultures. Nodular lesions on the arms and legs developed into abscesses, and growth of $S$. marcessens was observed in the wound cultures collected from these sites. The patient was started on meropenem treatment again, with local care and topical fucidic acid were applied to the abscesses. The skin lesions healed completely by day 20 after hospitalization, and chemotherapy was continued

Case 2- A 9-month-old female patient was diagnosed with high-risk ALL at our pediatric hematology clinic, and started on the treatment according to the St. Jude Total XV chemotherapy protocol. A CVC was implanted on the 10th day of the hospitalization, and neutropenic sepsis developed on day 15. The patient was started on $60 \mathrm{mg} / \mathrm{kg}$ of piperacillin-tazobactam and $15 \mathrm{mg} / \mathrm{kg}$ of amikacin treatment. $S$. marcessens growth was observed in both CVC and peripheral blood cultures. Piperacillintazobactam treatment was discontinued 2 days after the patient presented with antibiotic-resistant fever, and $90 \mathrm{mg} / \mathrm{kg}$ of meropenem treatment was started.

On the third day of the treatment, vesicular lesions started to appear on the dorsum of the hand and the body, and spread to the scalp and across the entire body the next day (Figure 1a, b). The lesions were not itchy, and mucosal spread was not observed.

Given the resemblance of the lesions to those seen in VZV infections, a sample was sent to test for VZV IgM, and acyclovir $(30 \mathrm{mg} / \mathrm{kg} / \mathrm{d})$ treatment was started. Her skin lesions diminished and healed within three days. Besides, the patient's fever began to drop down on the second day of meropenem treatment. Acyclovir treatment was discontinued after negative $\mathrm{VZV}$ IgM test results were obtained. CVC catheter was removed after S. marcessens growth on catheter was twice than that observed in the blood culture. Meropenem treatment was discontinued on the 15 . day of hospitalization at the time when microorganism growth in the peripheral blood culture was not observed, however chemotherapy was maintained.

\section{DISCUSSION}

According to the literature, skin manifestations are rarely seen in $S$. marcessens infections, and they are mostly observed in immunosuppressed adult patients. Skin lesions that develop during serratia infections are classified in two groups. The lesions start as acute cellulitis or abscesses, and may turn into ulcers or even severe necrotizing fasciitis and may take on a chronic form. On rare occasions, granulomatous lesions that start as nodules have been observed in the chronic form $[2,3,4]$.

Skin manifestations related to Serratia infections in pediatric cases are rarely reported, although Garcia et al. reported a case of a 10-year-old child diagnosed with $S$. marcessens, who had skin manifestations in the form of erythematous plaques on 

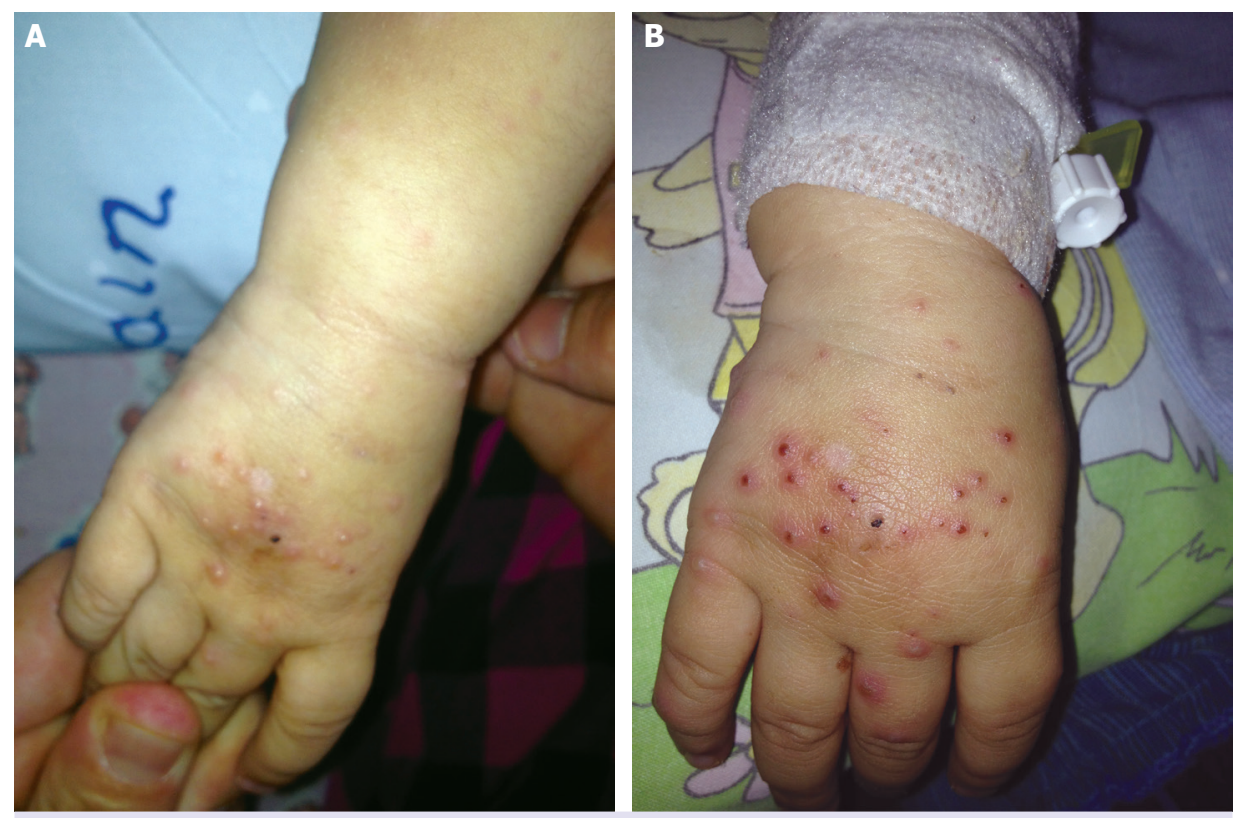

FIGURE 1. (A) Papillovesicular lesions on the dorsum of the hand. (B) Erythematous macules, fluid filled vesicles and crusting lesions on the dorsum of the hand.

an upper extremity [5].

Both cases discussed in this study were suffering from acute leukemia, and they were not in remission. On the third day of infection skin rashes in the form of vesiculobullous lesions emerged. Since the skin rashes resembled lesions of $\mathrm{VZV}$ infections and the patients had been diagnosed with acute unremitting leukemia, acyclovir treatment was initiated. In the pediatric age group, VZV infections are transmitted via airway or through direct contact. Rashes are firstly seen on the scalp, and from there they spread over the face and the body. VZV infections start as erythematous macules, and later turn into vesicles; and rashes may be detected in the same region in different phases. In a healthy person, rashes tend to continue until day $7 . \mathrm{VZV}$ infections are presented with highly severe complications, and can be fatal in patients with hematological malignancies, so it is recommended that intravenous acyclovir treatment be started as soon as suspected lesions are observed [6].

In our cases, the rashes initially emerged on the dorsum of the hand and body, and spread to the scalp. As new lesions started to appear, worms started to emerge from other areas. Unlike VZV infections, the lesions were not itchy and mucosal spread was not seen. The mean recovery time for rashes was 2-5 days. Both had a CVC in situ, and
S. marcessens growth was observed in both of their peripheral and CVC blood cultures. The VZV IgM results in both cases turned out to be negative, and so it was deduced that the patients' rashes were associated with a $S$. marcessens infection.

However, in immunocompromised patients, VZV antibody test may be false negative [6]. $\mathrm{Pa}$ tients must be followed closely. In our case, acyclovir therapy was discontinued because the serology was negative for VZV and the patients' lesions began to lessen rapidly after meropenem treatment was initiated. Also, Enterovirus can be the cause of vesicular rash in immunocompromised patients [7] However, we examined the oral cavity and couldn't find any lesions, so it was not considered as an Enteroviral infection.

A variety of skin rashes due to drug reactions can be seen. Most frequently antibiotics and anti-inflammatory drugs are causing drug-induced rashes [8]. In our cases, skin rashes began to emerge after meropenem treatment. However, meropenem was previously used in our patients without development of allergic reactions. In addition, the lessions were not itchy and disappeared with meropenem treatment. Therefore, skin rashes were not considered as manifestations of drug allergy. 
In the first case, the rashes started in the vesicular form located on the arms and legs turning into painful nodular lesions with time. The disease of the patient followed a course similar to the cases of Serratia-infection reported in the literature [2]. Some of these nodules developed into abscesses which were drained.

Maybe, this vesicular rash can be considered as an early sign of nodular lesions. However, these nodular lesions were not observed in our second case.

Serratia infections are more frequently observed in cases with immune deficiencies and may have diverse disease courses. Early diagnosis and treatment are vital in cases with both Serratia and VZV infections, as both diseases can be fatal.

Based on the absence of similar case reports in the literature, we report these two cases to emphasize that: i) $S$. marcessens infections can also lead to development of a papillovesicular rash similar to that seen in VZV infections, ii) S. marcessens infection should be considered, when papillovesicular rashes are observed in immunosuppressive patients with an implanted CVC in situ.

Conflict of Interest Statement: None of the authors of this paper has a conflict of interest, including spesific financial interests, relationships, and/or affilitions relevant to the subject matter or materials included.
Financial Disclosure: The authors declared that this study has received no financial support.

\section{REFERENCES}

1. Khanna A, Khanna M, Aggarwal A. Serratia marcescens- a rare opportunistic nosocomial pathogen and measures to limit its spread in hospitalized patients. J Clin Diagn Res 2013;7:243-6.

2. Giráldez P, Mayo E, Pavón P, Losada A. Skin infection due to Serratia marcescens in an immunocompetent patient. [Article in Spanish] Actas Dermosifiliogr 2011;102:236-7. [Abstract] CrossRef

3. João AM, Serrano PN, Cachão MP, Bártolo EA, Brandão FM. Recurrent Serratia marcescens cutaneous infection manifesting as painful nodules and ulcers. J Am Acad Dermatol 2008;58(2 Suppl):55-7. CrossRef

4. Langrock ML, Linde HJ, Landthaler M, Karrer S. Leg ulcers and abscesses caused by Serratia marcescens. Eur J Dermatol 2008;18:705-7.

5. García FR, Paz RC, González RS, Ruiz ES, Martín-Neda FG, Rodríguez MS, et al. Cutaneous infection caused by Serratia marcescens in a child. J Am Acad Dermatol 2006;55:357-8. CrossRef

6. Mattiuzzi GN, Cortes JE, Talpaz M, Reuben J, Rios MB, Shan J, et al. Development of Varicella-Zoster virus infection in patients with chronic myelogenous leukemia treated with imatinib mesylate. Clin Cancer Res 2003;9:976-80.

7. Karadağ Öncel E, Narz I, Özsürekçi Y, Korukluoğlu G, Cengiz AB, Ceyhan M. Demographic and Clinical Findings in Children with Enteroviral Infection Outbreak. J Pediatr Inf 2013;7:97-101.

8. Dincer D. Drug reactions in Dermatology. Turk J Dermatol 2013;7:179-84. 